\title{
Detection and characterization of composite real-life damage by the TSR-polynomial coefficients RGB-projection technique
}

\author{
by J.-M. Roche* and D. Balageas** \\ * ONERA, Composite Materials and Structures Dept., BP72, 92322 Châtillon cedex, France, jmroche@onera.fr \\ **Univ. Bordeaux, I2M, TREFLE Dept., UMR 5295, F-33400 Talence, France, daniel.balageas@u-bordeaux.fr
}

\begin{abstract}
The Thermographic Signal Reconstruction (TSR) techniques, whether based on the logarithmic polynomial derivatives or on the composite images built from RGB projections of polynomial coefficients, have been proven efficient to detect calibrated defects. The present paper illustrates the relevance of the RGB projection approach for the inspection of composite materials and structures with actual real-life damage such as manufacturing and impact-induced defects. For all studied cases, RGB-composite images are used to identify the delaminated zones and the possibility to further exploit them into actual depth mappings, which could be referred as "thermal D-scans", is discussed.
\end{abstract}

\section{Introduction}

The efficiency of the Thermographic Signal Reconstruction (TSR) technique applied to pulse thermography to improve the detection of defects is unquestionable [1-4]. A recent study has shown that the images of the logarithmic polynomial coefficients could also be successfully used to build a unique composite image, by an RGB projection, which significantly simplifies the inspection carried out by the operator [5-8]. Moreover, based on these first results, it was noticed that the colors of the RGB-composite image were directly linked to the variations of depths of the defects. However, this approach has only been validated for the detection of calibrated defects, of known size and depth. The present work aims at evaluating the relevance of this processing technique for the detection and characterization of defects resulting from real-life damage in composite materials, and thus to contribute to the field of damage tolerance.

As far as damage tolerance of composite materials is concerned, two distinct types of defects are commonly considered: defects initially created during the manufacturing process, and defects induced by accidental events, such as impacts, or by recurring mechanical loadings, such as fatigue. The present paper illustrates the contribution of the TSRpolynomial coefficients RGB projection technique for the detection and characterization of initial defects and of impact damage, for various composite materials and structures. The opportunity to use TSR data to produce a full depthmapping of the detected defects, which could be called "thermal D-scan", is studied and commented on as well.

\section{Use of the TSR-coefficient RGB-composite images: from detection to depth-mapping}

\subsection{Recall of the TSR processing techniques}

The TSR processing technique was initially proposed to improve the detection of defects by pulse thermography [1-4]. The main purpose was to emphasize the thermal contrasts generated by the defects inside the inspected material, by considering the images of the logarithmic derivatives of the temperature variation $\Delta T$. To do so, a fitting of the logarithm of $\Delta T$ is performed by a polynomial of degree $n$ :

$$
\ln (\Delta T)=a_{0}+a_{1} \ln (t)+a_{2}[\ln (t)]^{2}+\ldots+a_{n}[\ln (t)]^{n}
$$

which then enables the computation of the derivatives with a limited increase of temporal noise. It was shown that, when detection is concerned, the first and second logarithmic derivatives did provide better defect mappings than other postprocessing techniques such as principal component thermography, pulse-phase thermography or higher-order statistics [6]. However, two major drawbacks can still be noted: it is necessary to observe the whole sequence of images, which is quite time-consuming, therefore not practically usable by an operator during manufacturing process or maintenance; plus, the derivative images give no information regarding the depths of the detected defects, although this unexplored possibility should not be completely discarded [9].

In order to get around this first issue, it was proposed in a few studies to analyze the small series of the $n+1$ coefficient images $\left\{a_{0}(i, \jmath) ; \ldots ; a_{n}(i, \jmath)\right\},(i, \jmath)$ being the pixel space coordinates, instead of the whole sequence of images [10-12]. The data reduction is significant, from a few thousand images to a dozen images at most. Yet, since time information is completely lost, these coefficient images cannot be linked to any depths. This is why a further step was proposed, consisting in the synthesis of a unique composite image, in color, obtained by the projection of three coefficient images on an RGB (Red, Green, Blue) basis [5-8]. Not only is the image unique, which makes the inspection 
operation almost instantaneous, but it also appears that every cluster of similar thermograms is associated with one given color: all defects embedded at a given depth, generating local discontinuities at a given time, are associated with the same color: different colors mean different depths. Yet, if a dependency of the colors with the depths is shown, the absolute values of the depths remain unknown.

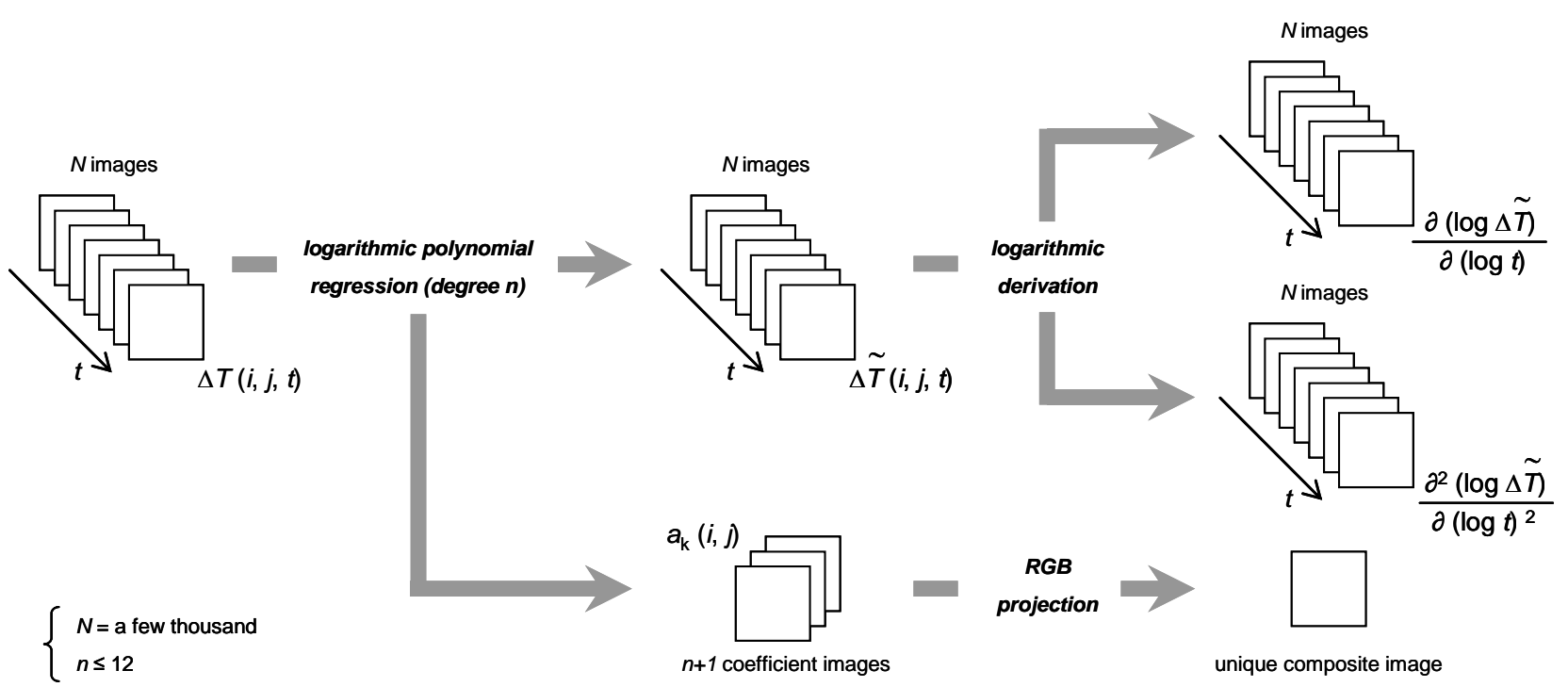

Fig. 1. TSR processing techniques used for defect detection: logarithmic derivations (standard approach) and $R G B$ projection of coefficient images (ONERA approach).

\subsection{Thermal depth-mapping: theoretical approach}

Ever since stimulated infrared thermography was used for NDT, works have been carried out with the purpose of not only detecting defects, but also quantitatively characterizing them, starting with assessing their depths. The setting of a thermal "3D mapping" of defects, similar to an ultrasound D-scan, is thus a great stake. In the eighties and nineties, several authors proposed to build various types of images:

- "timegrams": such images are time-graduated with the times associated to given levels of relative thermal contrast (maximum contrast for instance). Once isochronous slices are realized, tomographies of the sample can be provided, assuming that the defect resistances are very high or identical. These images were referred to as "tomograms" by Vavilov [13]. More thorough discussions about the concept of thermal tomography were set in recent articles [14, 15].

- "defect-depth images" or "depthgrams": these images are produced from timegrams, using an expression correlating a given contrast and its time of occurrence to a defect depth $z_{\mathrm{d}}$. This approach has been followed in various papers [16-18], in which the apparent effusivity is considered instead of the temperature variation $\Delta T$. Eventually, the expression given by equation (2) was admitted to lead to errors lower than $10 \%$, as long as defects are not too close to the rear-face of the sample and have an extent of several times their depths (to limit 3D effects).

$z_{\mathrm{d}}=\sqrt{\alpha_{\mathrm{z}} t_{\max }}\left(\frac{\Delta T_{\mathrm{s}}}{\Delta T_{\max }}\right)^{n}=\frac{\sqrt{\alpha_{\mathrm{z}} t_{\max }}}{\left(1+C r_{\max }\right)^{n}}$

where $\alpha_{z}$ is the through-thickness thermal diffusivity, $C r_{\max }$ the maximum relative contrast between the temperature variation $\Delta T_{\max }$ at the defect location and the temperature variation $\Delta T_{\mathrm{s}}$ in the sound zone, occurring at time $t_{\max }$.

The value of the exponent $n$, that can be found equal to 0.95 or 0.97 depending on the reference, can just be fixed to 1, leading to a simpler expression:

$z_{\mathrm{d}}=\sqrt{\alpha_{\mathrm{z}} t_{\max }} \frac{\Delta T_{\mathrm{s}}}{\Delta T_{\max }}=\frac{\sqrt{\alpha_{\mathrm{z}} t_{\max }}}{1+C r_{\max }}$

A similar expression, not recalled here, based on the half-maximum contrast, has also been proposed.

To mitigate the problematic influence of the rear-face and of the finite extent of the defects, the use of the emerging contrast to identify depths (as well as thermal resistances) appeared at the beginning of the nineties. This approach, known as the early detection method, led to the use of expressions of varied complexity. The first one, reminded by equation (4), was proposed by Bontaz et al. [18, 19]:

$z_{\mathrm{d}}=\sqrt{2 \pi \alpha_{\mathrm{z}} t_{0}}$

where $t_{0}$ is the time of emergence of the thermal contrast. 
Given its simplicity, this expression is quite convenient to use. However, it suffers from three weaknesses: first, no criteria is defined to estimate the emerging contrast; second, the determination of $t_{0}$ gets very tricky as soon as noisy signals are to be dealt with; finally, the coefficient $2 \pi$ comes from an arbitrary value of the defect Fourier number Fo $=1 / 2 \pi=0.159$ and of the emerging contrast equal to $1 / \pi=0.318$ for a highly resistive defect.

This is why some improvements have been proposed by Krapez et al. [20, 21], eventually completed by the linear extrapolation to zero-contrast [4, 22]:

$z_{\mathrm{d}}=\lim _{C r \rightarrow 0}\left(\sqrt{\ln \left(\frac{2}{C r(t)}\right) \alpha_{\mathrm{z}} t}\right)$

Any of those approaches can be used to evaluate either the thickness $L$ of the inspected sample, as long as its thermal diffusivity is known, or reciprocally the thermal diffusivity, when the thickness can be measured. Indeed, in comparison to the theoretical semi-infinite medium solution, the rear-face creates a contrast which is similar to the one induced by a defect of infinite thermal resistance (Figure 2). Then, the application of the simplified formula (4) leads to a relation of proportionality between the depth of a given defect $z_{\mathrm{d}}$ and the thickness $L$ :

$z_{\mathrm{d}}=L \sqrt{\frac{t_{\mathrm{d}}}{t_{\mathrm{L}}}}$

where $t_{\mathrm{d}}$ and $t_{\mathrm{L}}$ are the emerging times of the contrasts respectively induced by the defect and by the rear-face.

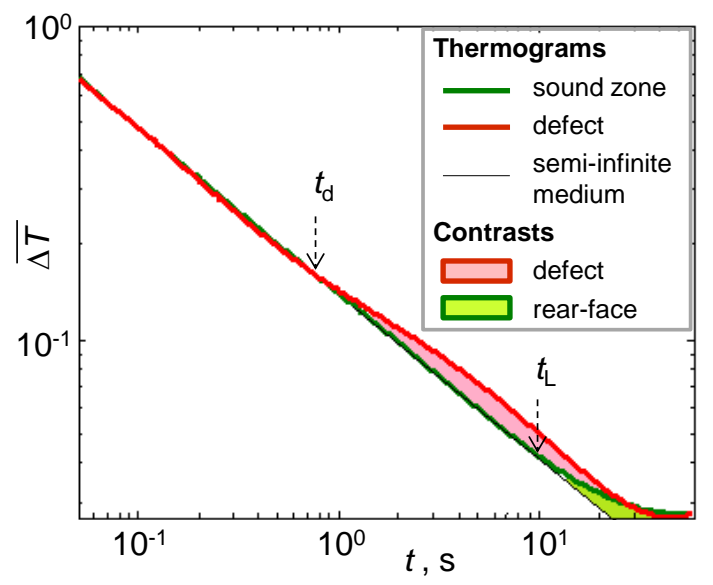

Fig. 2. Theoretical time-evolution of the normalized temperature decrease for a sound zone and a defective zone in a homogeneous material. Contrasts generated by the defect and by the rear-face (compared to the semi-infinite medium).

\subsection{Proposition of a TSR-based approach for defect imaging and depth mapping}

In the present paper, the followed philosophy is the one of "depthgrams", using the simple expression given by equation (6) to estimate the depths of the defects identified in the RGB-composite images. The thicknesses of all inspected samples are measured and supposed to be homogeneous unless specified otherwise. The stake of this work is quite obvious: based on these previous considerations, it seems that the production a thermal $D$-scan requires a timeresolved analysis of the contrast evolution in the full field of view of the camera, which means the observation of the full sequence of infrared images. Consequently, it would be a very promising perspective if depth-mapping could be achieved thanks to the RGB-composite image, which is almost automatically built.

The relevance of this approach is challenged for real-life damage in composite materials and structures. To generate a thermal D-scan, a two-step procedure is proposed:

- application of the TSR processing tools to the raw thermal data and production of a unique RGB-composite image to identify all inhomogeneities in the inspected coupon or structure;

- assessment of the defect depths, using equation (6), based on the associated fitted thermograms averaged over a small number of pixels picked in the defective areas.

Two distinct objectives are aimed at with this approach. The first step, for detection purpose only, can be validated by comparing the RGB-composite image to the classic TSR logarithmic images. The second step, however, for which the estimation of the defect depths is aimed at, could only be rigorously validated by comparing the results to ultrasound D-scans or, even better, to micrographic cross-sections. These data are unfortunately missing in most of the cases, because immersion ultrasonics testing is forbidden for some samples (to avoid humidity aging of the resins), as well as cross-sections cuttings. This lead might be followed in future works, for certain appropriate materials. For now, 
the only validation that can be done is the comparison between two front-face pulse thermography experiments successively performed on both faces of the sample: for one given defect, as long as it is not overlapped with another one, the sum of the depths estimated from both experiments should be equal to the total thickness.

\section{Experimental setup and tested composite coupons and structures}

All results presented in the following sections were acquired from works carried out in ONERA. The front-face pulse thermography experiments are performed using two Elinchrom flash lamps delivering a pulse of a total energy of $6 \mathrm{~kJ}$ in $4 \mathrm{~ms}$. The lamps are equipped with plexiglas filters, so that the residual infrared radiations have a limited effect. $A$ middle-wave infrared camera (FLIR X6540-SC), covering a [3 - 5] $\mu \mathrm{m}$ wavelength range, with a 20 mK noise equivalent temperature difference (NETD), and a spatial resolution of $640 \times 512$ pixels, is used to record the images. The recording duration is chosen depending on the thermal properties and the thicknesses of the inspected materials. Once raw thermal data is acquired, the full TSR-based procedure, detailed in Section 2, is carried out.

Several types of composites are studied. Section 4 is dedicated to the quantitative non-destructive evaluation (NDE) of manufacturing defects in a carbon/silicone laminate composite and in a woven oxide/oxide ceramic matrix composite. In Section 5, impact-induced damage in carbon/epoxy laminate composites, from the basic plate to more complex structures such as a stiffened panel and a honeycomb sandwich panel, is studied.

\section{Detection and characterization of manufacturing defects}

For both coupons studied in this section, a non-intrusive inspection was required: because of the components of the composite material, NDE tests in immersion, such as ultrasound scans, were not an option. Pulse thermography was a natural candidate.

\subsection{Inspection of a carbon/silicone laminate composite coupon}

The first studied coupon is a $300 \times 300 \mathrm{~mm}^{2}$ coupon, $6 \mathrm{~mm}$ thick, with a silicone-based resin and carbon fibers. Its manufacturing process is constituted of several steps, including injection moulding, pyrolysis and infusion. The pyrolysis step is the problematic one: a slight increase of the global thickness is measured, which lets the operator suspect the presence of defects such as delamination.

Given the thickness of the sample, thermal data is acquired over $120 \mathrm{~s}$, at a $100 \mathrm{~Hz}$ frequency. The second post-flash image, in which the flash distribution is more homogeneous than in the first one, is used for the normalization operation. The classic TSR technique is then carried out, with a logarithmic polynomial of degree 7 . The choice of this degree was recommended in a previous study for the inspection of a composite plate with embedded calibrated defects [6]. The time-evolutions of the first and second logarithmic derivatives in identified defective zones and in the sound zone are displayed in Figures 3 and 4, as well as the images associated with the times of maximum contrasts (the objective being to get the highest contrasted images, so that detection of defects can be as easy as possible).
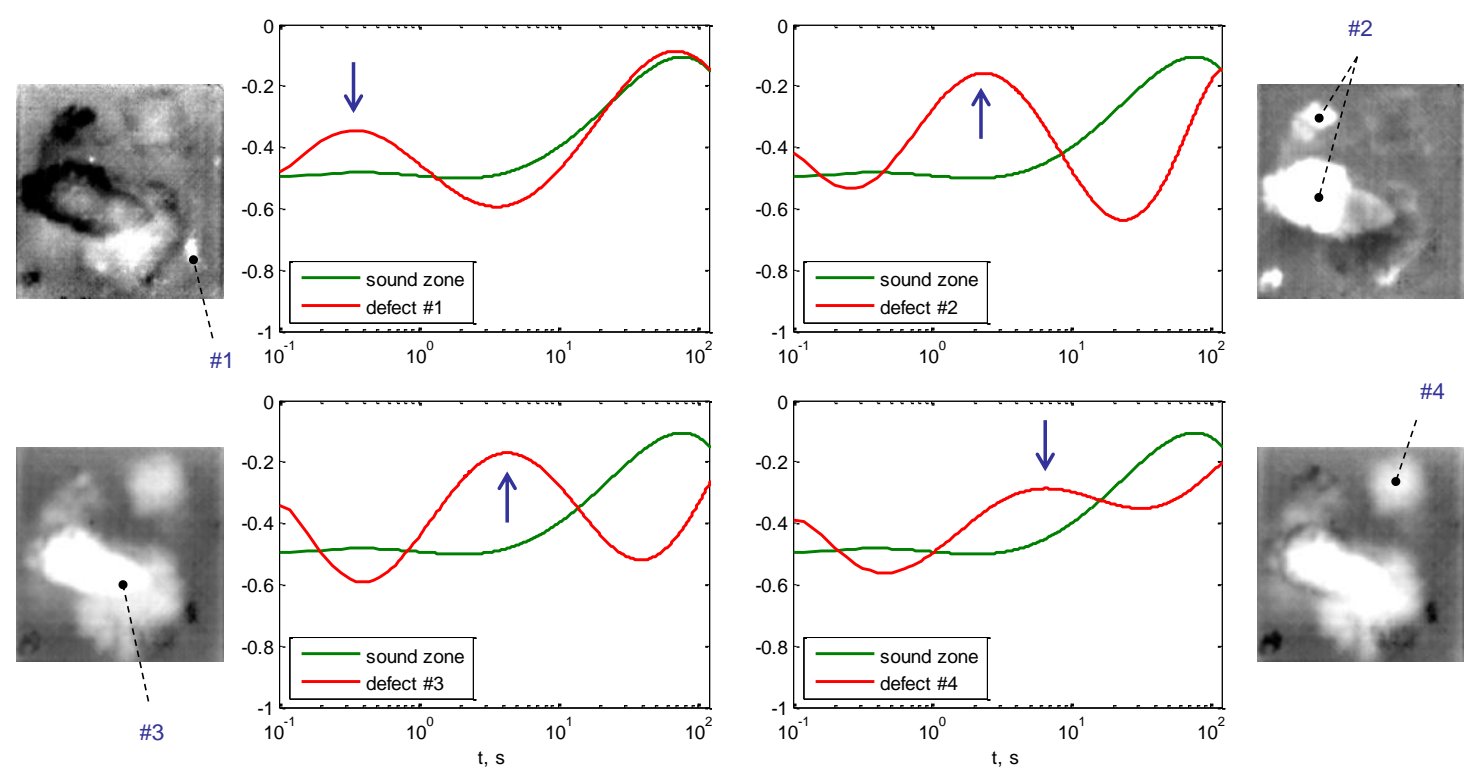

Fig. 3. Time-evolution of the $1^{\text {st }}$ logarithmic derivatives in defective zones and in the sound zone. Matching images, selected at the times of maximal contrasts. TSR treatment, polynomial of degree 7. 

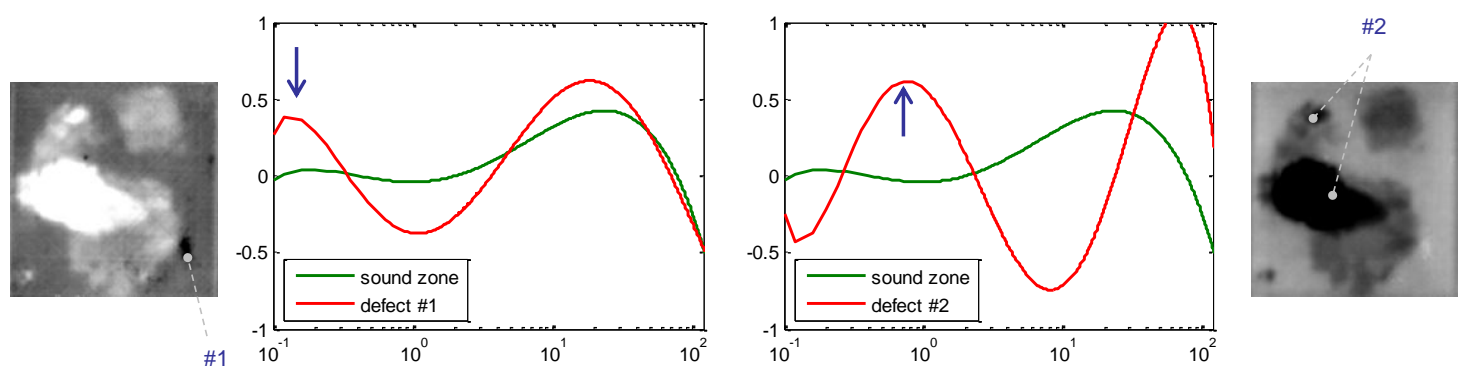

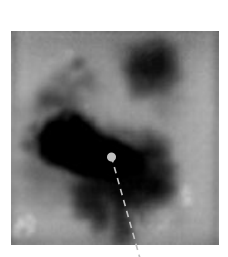

\#3

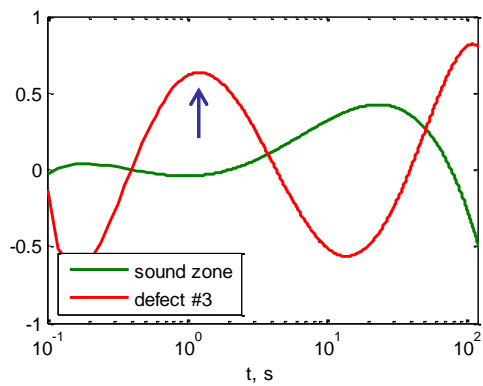

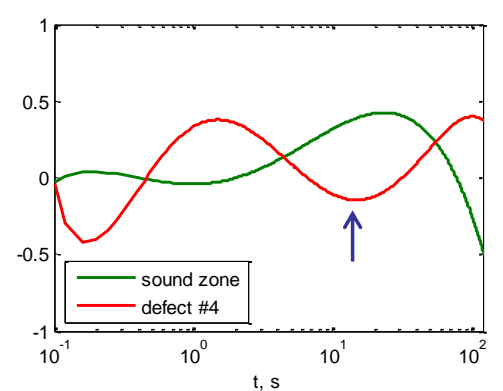

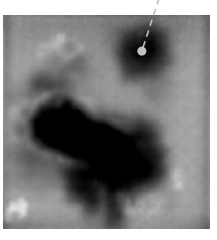

Fig. 4. Time-evolution of the $2^{\text {nd }}$ logarithmic derivatives in defective zones and in the sound zone. Matching images, selected at the times of maximal contrasts. TSR treatment, polynomial of degree 7.

As expected, the contrasts are emphasized, both on $1^{\text {st }}$ and $2^{\text {nd }}$ derivative images, which enables a consistent "homogeneity inspection" of the coupon: several delaminated zones are spotted, some of which appearing in different areas of the coupon but at the same depth (second-level defects in Fig. 3 and 4). However, the process of selecting the best images is time-consuming, and no information on the depths of these defects can be given.

The eight coefficient images are then studied, so that the three best ones, based on defect detectivity purposes, are selected. Two trios of monomials are identified: the $\{5,2,3\}$ one and the $\{2,5,7\}$ one. The resulting RGB-composite images are given in Figure 5. If the colors differ from one image to another, the exact same defective zones are identified, which is a good point as far as the consistency of the method is concerned. The other very satisfactory point is that all the delaminated zones pointed out with the analysis of the logarithmic images are seen in the RGB-composite images, which reinforces the relevance of the present approach for non-destructive inspection.
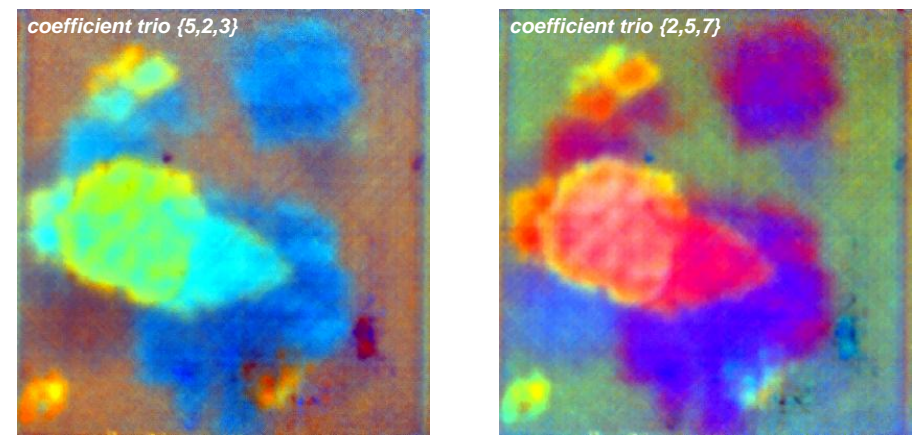

Fig. 5. TSR-coefficient RGB-composite images, produced from two trios: $\{5,2,3\}$ (left) and $\{2,5,7\}$ (right).

Based on arbitrary eye-sensitivity criteria, the $\{5,2,3\}$ trio is chosen. Seven color shapes are identified: one for the sound zone, characterized by the rear-face of the coupon; six others for delaminated zones. For each and every one of these zones, normalized fitted temperature decreases are plotted (Figure 6) and the times of emerging contrasts $t_{\mathrm{i}}$ are estimated. It should be noted that, in order to avoid non-physical contrasts due to inaccurate fitting in the early times, logarithmic polynomials of degree 10, instead of 7, are used. As explained in Section 2, the thickness of the coupon can be checked from the difference with the semi-infinite homogeneous temperature behavior (linear, with a $-1 / 2$ slope, in a log-log scale), and the depths of the delaminated zones can be estimated with the expression of equation (6).

Once the depths are estimated, the matching scale can be associated to the RGB-composite image, which ultimately provides a thermal D-scan (Figure 6). It is quite satisfactory to see that the rear-face of the coupon is clearly seen, despite its thickness $(6 \mathrm{~mm})$, and that delaminated zones are detected up to a depth of $4 \mathrm{~mm}$. 
The very same processing procedure is applied to the thermal data acquired from the pulse thermography experiment on the opposite face of the coupon. Once again, the bottom is detected and the global thickness of $6 \mathrm{~mm}$ checked; as for delaminated zones, the deepest one is found to be at a $5 \mathrm{~mm}$ depth, which is quite spectacular for thermography (Fig. 7). Both thermal D-scans are compatible: delaminated zones found at depths of respectively $1.8 \mathrm{~mm}$, $3.3 \mathrm{~mm}$ and $4.1 \mathrm{~mm}$ in the first image turn up at depths of $4.4 \mathrm{~mm}, 3.0 \mathrm{~mm}$ and $1.8 \mathrm{~mm}$ in the second image. The sum of the depths leads to values close to the actual thickness (respectively $6.3 \mathrm{~mm}, 6.3 \mathrm{~mm}$ and $5.9 \mathrm{~mm}$ ), which definitely validates the quantitative content of the RGB-composite image, and consequently the depth mapping.
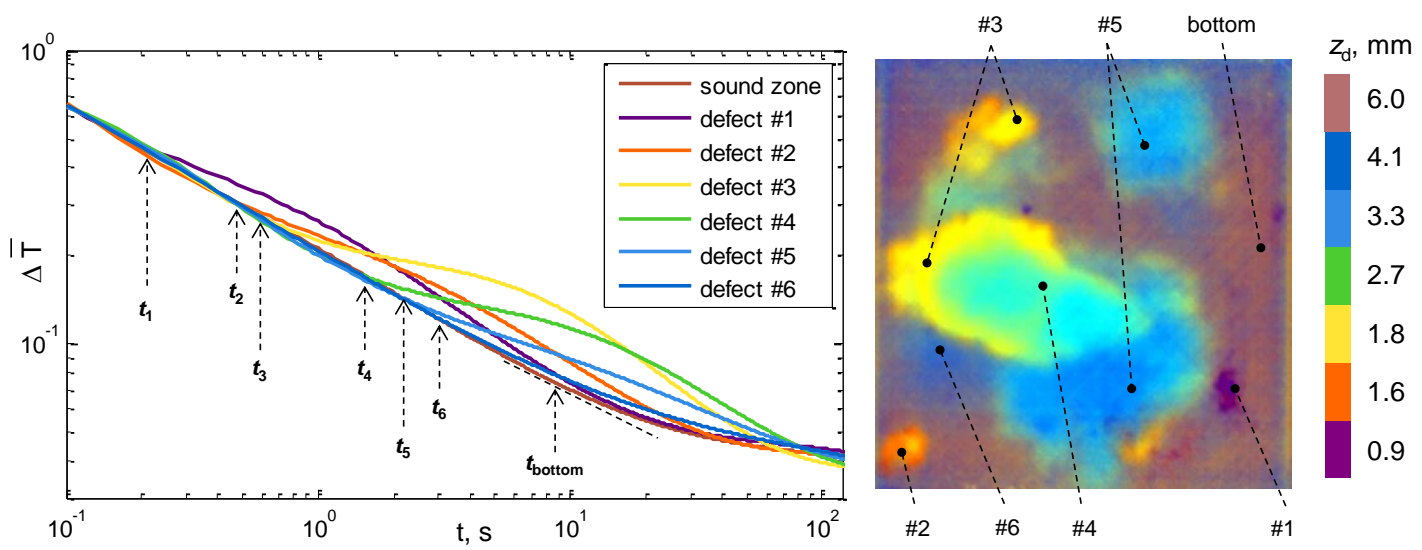

Fig. 6. Generation of a thermal D-scan, using the RGB-composite image (TSR degree 7), and the depth values estimated from the fitted normalized temperature decreases (TSR degree 10). $300 \times 300 \mathrm{~mm}^{2}$ carbon/silicone coupon.
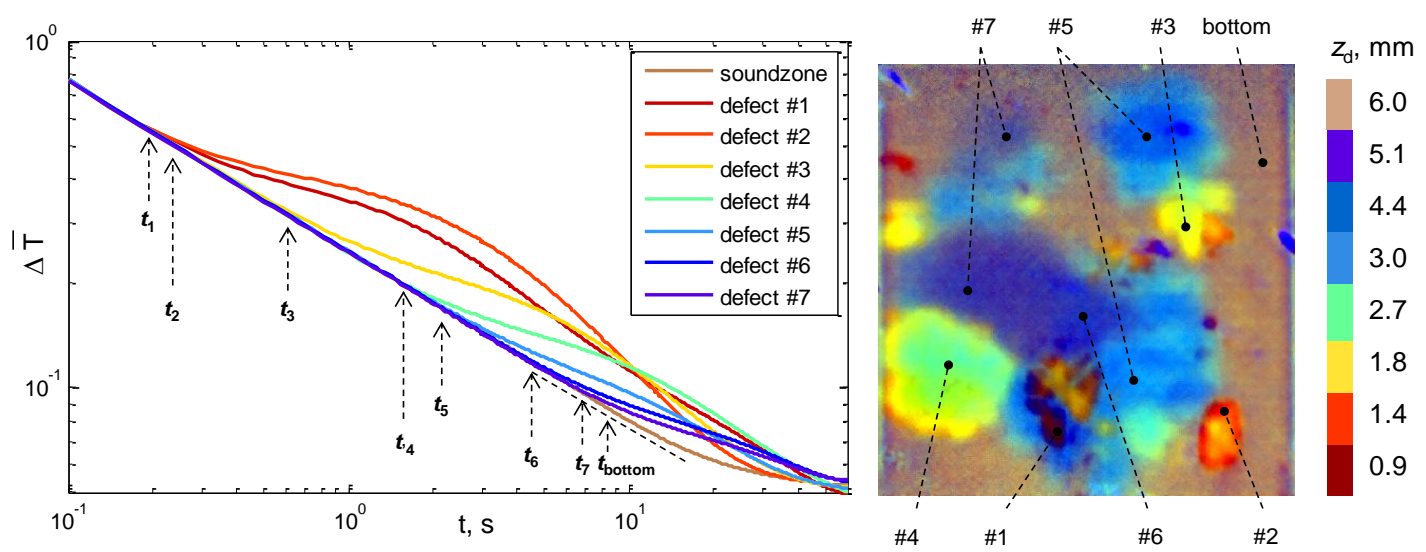

Fig. 7. Opposite face RGB-composite thermal D-scan, flipped over across vertical axis. Same procedure as in Fig. 6.

The application of the more thorough procedure of "early detection" could give even more credit to those thermal D-scans, since it would bring more confidence in the calculated depth values. This possibility is likely to be studied in future works.

\subsection{Inspection of two-dimensional woven oxide/oxide composite coupon}

The second studied coupon is a $150 \times 150 \mathrm{~mm}^{2} 2 \mathrm{D}$ woven oxide/oxide composite. Its thickness is not strictly constant from one zone to another: measurements show that it varies between $2.25 \mathrm{~mm}$ and $2.45 \mathrm{~mm}$. The manufacturing process of these ceramic matrix composites is still being optimized, the main difficulty being to respect the $25 \%$ aimed porosity rate, and avoiding edge delamination and macroporosities at the same time. Once again, ultrasound scan should be avoided since the material is porous, and priority is given to front-face pulse thermography.

Since the coupon is white, its surface is blackened with a peelable paint. Thermal data is acquired over $30 \mathrm{~s}$, at a $100 \mathrm{~Hz}$ frequency. Figure 8 displays the results of the TSR-based processing procedure: the time-evolution of the TSRfitted normalized temperature decrease, and the RGB-composite image.

If the inhomogeneities of the coupon are indeed detected (a few locally delaminated zones and one large macroporosity), with emphasized contrasts, the quantitative treatment cannot be fully followed through. The rear-face is detected at a time of about $0.3 \mathrm{~s}$. Assuming a thermal diffusivity of $3 \mathrm{~mm}^{2} / \mathrm{s}$ (common value for these composites), the thickness of the coupon can be estimated at $2.38 \mathrm{~mm}$, which is in good agreement with the measured values. However, the variations of the normalized temperature in the defective zones cannot be used for depth estimation as proposed in 
Section 2. Two reasons preventing from using the expression of equation (6) can be given: first, the non-constant thickness of the coupon; second, the inhomogeneity of the porous material, leading to variations of density or/and heat capacity, which explains why the thermograms do not reach an identical and constant level at late times. Obviously, further studies dedicated to this type of composites are needed.
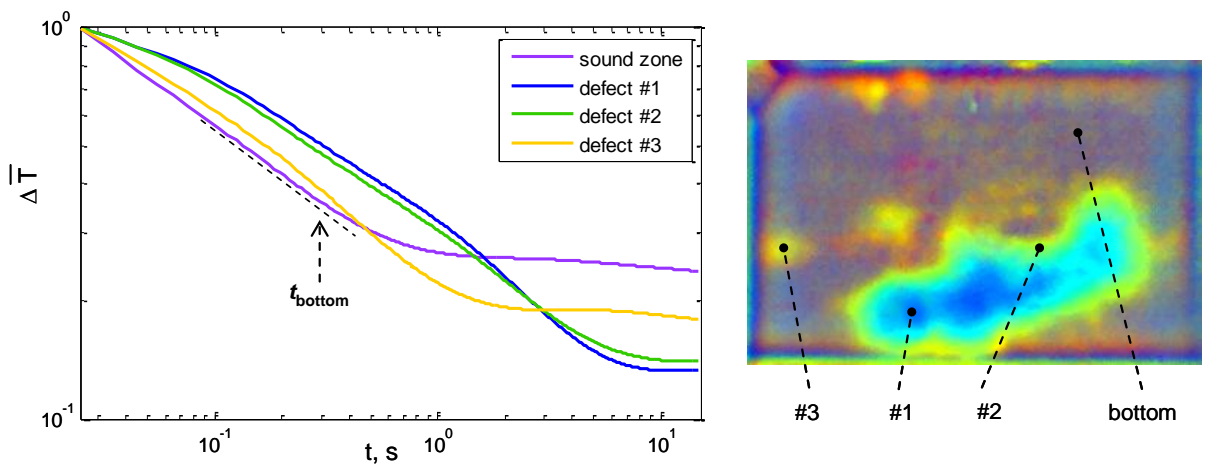

Fig. 8. RGB-composite image for a $150 \times 150 \mathrm{~mm}^{2}$ oxide/oxide coupon (TSR degree 7 , coefficient trio $\{2,3,4\}$ ). Illustration of the inhomogeneity of the material, which does not permit the depth mapping.

\section{Detection and characterization of impact-induced defects}

In this section, the TSR-based procedure is evaluated for the detection of accidental defects during maintenance operations. More specifically, low-velocity impact damage, which is another burning issue for the aeronautic industry, is studied. Once again, the rapidity of the image processing technique is essential.

Three cases, of increasing complexity, are presented: a small-dimension plate, a stiffened panel and a honeycomb sandwich panel. Carbon/epoxy laminate composites only are considered, which indicates that the nature of the defects to be detected should be delamination. Moreover, the thermal diffusivity is well-known, around $0.3 \mathrm{~mm} / \mathrm{s}$, which simplifies the choice of the acquisition parameters.

\subsection{Inspection of a carbon/epoxy laminate composite coupon}

The first coupon is a $150 \times 150 \mathrm{~mm}^{2}$ plate, $4.16 \mathrm{~mm}$ thick ( 16 plies of orientation $\left\{45^{\circ} / 90^{\circ} /-45^{\circ} / 0^{\circ}\right\}_{2 \mathrm{~s}}$ ), with an epoxy resin and carbon fibers. It is impacted by a $16 \mathrm{~mm}$ diameter hemispherical impactor, with an energy of $28 \mathrm{~J}$. Frontface pulse thermography experiments are carried out, for both faces of the plate, with a $100 \mathrm{~Hz}$ frequency, and the thermal data is acquired over $60 \mathrm{~s}$.

The RGB-composite images are displayed in Figure 9 (for the impacted side) and Figure 10 (for the opposite side). The trio of monomials $\{6,5,7\}$ is chosen in both cases. Delaminated areas are detected up to a depth of $2.3 \mathrm{~mm}$. The compatibility between both scans is not easy to be highlighted, since many delamination zones are detected at very shallow depths, on both sides, and often overlapping each other. However, delaminated zone \#4, detected at a $1.7 \mathrm{~mm}$ depth on the first thermal D-scan, matches with delaminated zone \#4, detected at a $2.3 \mathrm{~mm}$ depth on the opposite side thermal D-scan: the sum of those values is equal to $4 \mathrm{~mm}$, which is quite close to the total thickness of the plate.
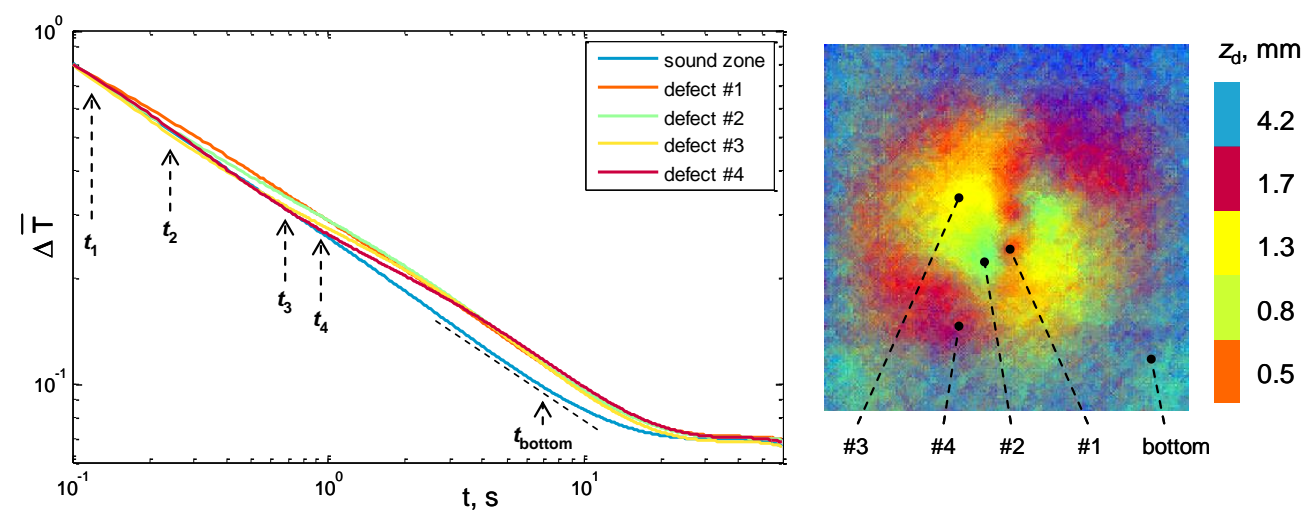

Fig. 9. Generation of a thermal D-scan, using the RGB-composite image (TSR degree 7, coefficient trio $\{6,5,7\})$, and the depth values estimated from the fitted normalized temperature decreases (TSR degree 10).

$150 \times 150 \mathrm{~mm}^{2}$ carbon/epoxy laminate plate, impacted face. Observation of a $100 \times 100 \mathrm{~mm}^{2}$ reduced zone. 

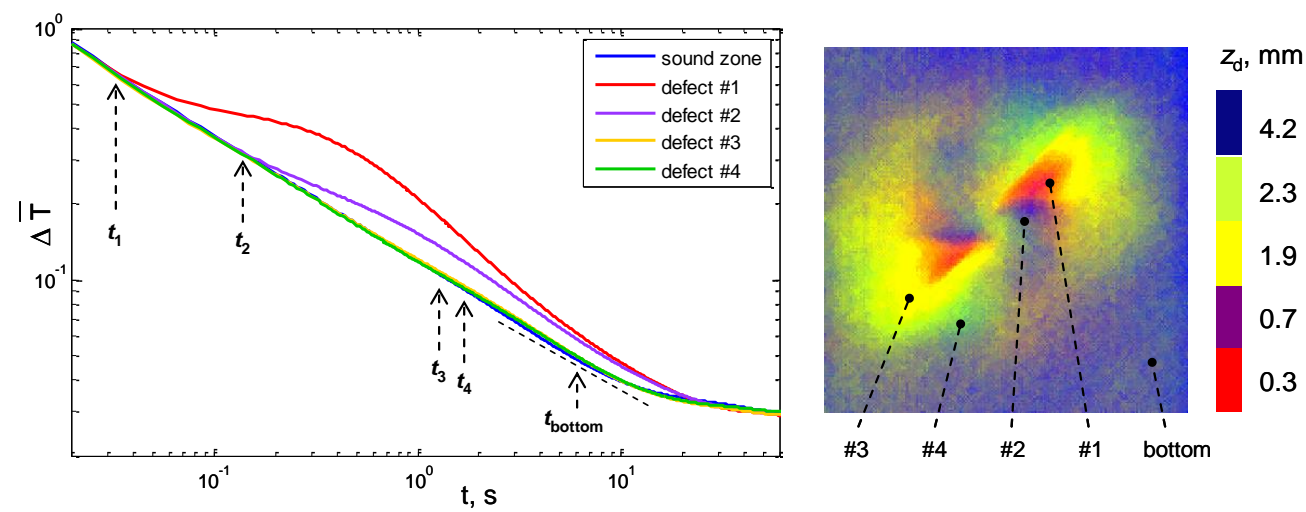

Fig. 10. Opposite face RGB-composite thermal D-scan, flipped over across vertical axis. Same procedure as in Fig. 9.

Further validation can be made by comparing the thermal D-scan of Figure 9 to the matching ultrasound Dscan, which was carried out for a previous study [24]. As illustrated by Figure 11, both scans are in good agreement: the surfaces of the delaminated area are almost identical, and the depths of the shallowest defects are quite close; however, non-negligible differences are found for the deepest defects. That observation is under on-going further investigation.
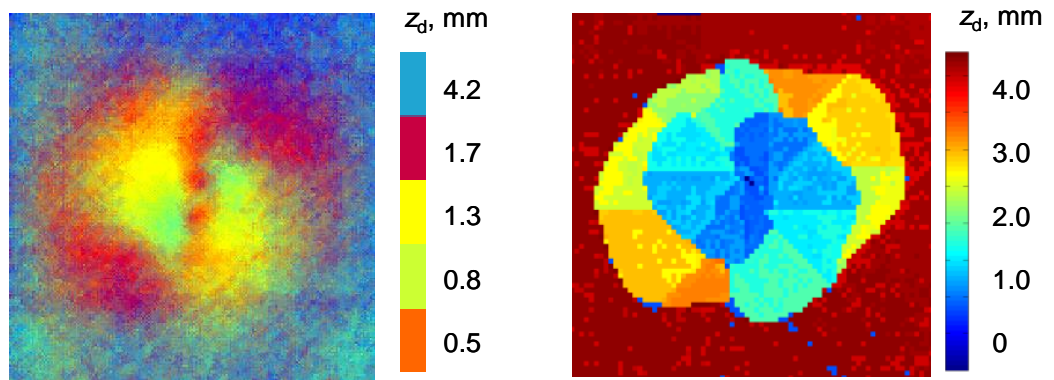

Fig. 11. Comparisons between the RGB-composite thermal D-scan (left) and the matching ultrasound D-scan (right).

\subsection{Inspection of a carbon/epoxy laminate composite stiffened panel}

The second case of study is an actual structure, a $675 \times 480 \mathrm{~mm}^{2}$ stiffened panel, $3.12 \mathrm{~mm}$ thick (12 plies of orientation $\left\{90 \%+45 \% 0^{\circ} / 0^{\circ} /-45^{\circ} / 90^{\circ}\right\}_{\mathrm{s}}$ ) in between the stiffeners and $4.20 \mathrm{~mm}$ thick (8 additional plies of orientation $\left.\left\{+45^{\circ} / 0^{\circ} /-45^{\circ} / 0^{\circ}\right\}_{\mathrm{s}}\right)$ on the stiffeners. Front-face pulse thermography experiments are carried out, before and after the impact, with a $100 \mathrm{~Hz}$ frequency, over $60 \mathrm{~s}$.

Figure 12 displays the RGB-composite image, produced from the monomials trio $\{6,5,4\}$, for the whole panel. Another advantage of this technique is pointed out: it can be applied, as it is, to the largest structures, with no additional time needed. The thermal D-scan identifies the thickness of the panel skin, which is found to be $3.1 \mathrm{~mm}$, and the thickness of the stiffeners base, which is estimated at $4.2 \mathrm{~mm}$. Another "inhomogeneity", associated with an orange color, appears: it indicates the position of the stiffeners (which of course are much thicker in the $z$ direction).

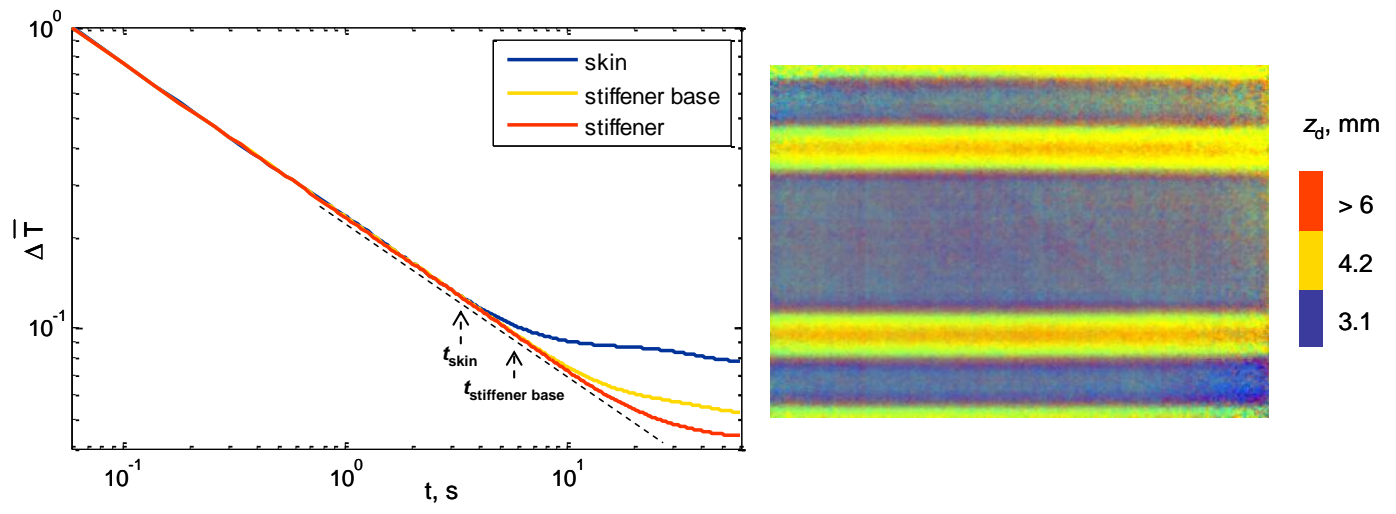

Fig. 12. Generation of a thermal $D$-scan, using the RGB-composite image (TSR degree 7 , coefficient trio $\{6,5,4\}$ ), and the depth values estimated from the fitted normalized temperature decreases (TSR degree 10). $675 \times 480 \mathrm{~mm}^{2}$ carbon/epoxy laminate stiffened panel, before impact. 
The thermal D-scan of the zone impacted at $22 \mathrm{~J}$, in between the stiffeners, with the $16 \mathrm{~mm}$ diameter hemispherical impactor, is displayed in Figure 13. The typical impact damage pattern is checked and delamination up to a depth of $1.5 \mathrm{~mm}$ depth is detected.
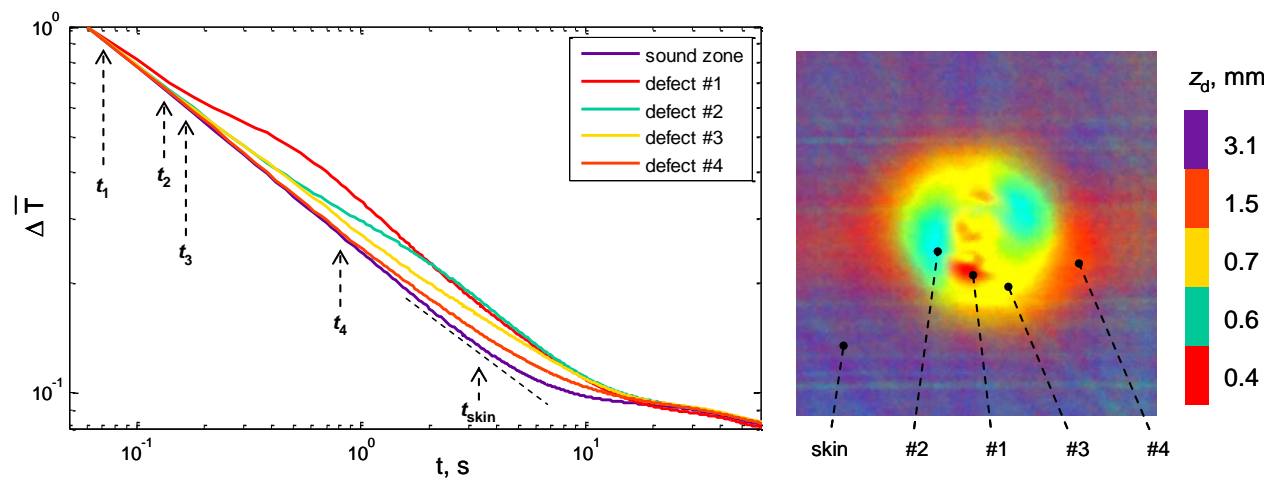

Fig. 13. Generation of a thermal D-scan, using the RGB-composite image (TSR degree 7 , coefficient trio $\{6,5,4\})$, and the depth values estimated from the fitted normalized temperature decreases (TSR degree 10).

$675 \times 480 \mathrm{~mm}^{2}$ carbon/epoxy laminate stiffened panel, impacted face. Observation of a $100 \times 100 \mathrm{~mm}^{2}$ reduced zone.

\subsection{Inspection of a carbon/epoxy laminate honeycomb sandwich panel}

The last studied sample is a $150 \times 150 \mathrm{~mm}^{2}$ honeycomb sandwich panel. The skin is a carbon/epoxy composite, $2.08 \mathrm{~mm}$ thick (8 plies); the honeycomb cell is aluminum made. The panel is impacted with a $40 \mathrm{~mm}$ diameter hemispherical impactor, at $4 \mathrm{~J}$. The front-face pulse thermography experiment is carried out with a $100 \mathrm{~Hz}$ frequency. Thermal data is acquired over $60 \mathrm{~s}$, processed as for the previous composite coupons, which leads to the coefficient RGB-composite thermal D-scan produced from the trio of monomials $\{3,7,5\}$ (Fig. 14). The geometrical honeycomb pattern is found, which makes it much harder to detect defects, since it is seen as periodic inhomogeneities, from a thermal point of view. The very same problem will have to be faced for the non-destructive quantitative inspection of woven composites. For the present sandwich panel, shallow delaminated areas, of depths lower than $1.2 \mathrm{~mm}$, are still detected and characterized with no particular difficulties.
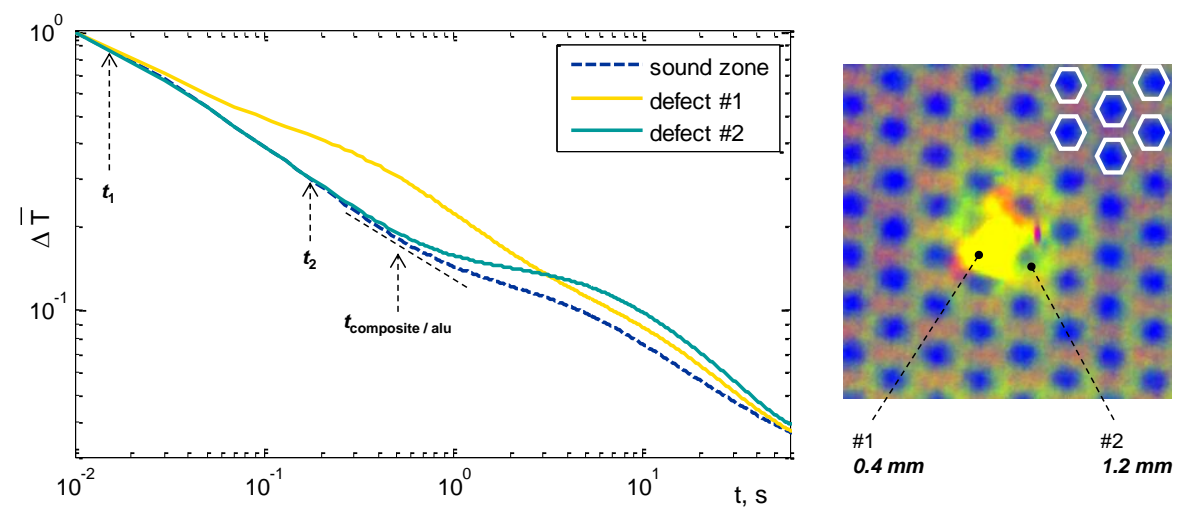

Fig. 14. Generation of a thermal D-scan, using the RGB-composite image (TSR degree 7 , coefficient trio $\{3,7,5\})$, and the depth values estimated from the fitted normalized temperature decreases (TSR degree 10).

$150 \times 150 \mathrm{~mm}^{2}$ carbon/epoxy laminate sandwich panel, impacted face. Observation of a $65 \times 65 \mathrm{~mm}^{2}$ reduced zone.

\section{Conclusions and prospects}

The first aim of the paper was to evaluate the relevance of the processing method based on the RGB projection of three TSR coefficient images into a unique composite image, initially proposed and validated for calibrated defects [6], for the detection and characterization of actual real-life damage. Manufacturing and impact-induced damage was considered, as well as different types of composite materials, from classic organic matrix laminates to woven ceramic matrix oxide/oxide composites, and different structures, from classic plates to a stiffened panel and a honeycomb sandwich panel. In all cases, the procedure has been validated: the RGB-composite image contains all information needed to detect the damaged areas.

As for a quantitative processing, the idea was to underline the specificity of the RGB-composite image, the colors of which are linked to depth variations, and to further exploit it into an actual depth mapping, which could be 
referred as "thermal D-scan". It was thus proposed to estimate the defect depths from the TSR-fitted normalized temperature decreases, using a simple analytic approximation, and to associate the found values with the colors of the RGB image. Apart from the inhomogeneous oxide/oxide plate, for which no thorough quantitative interpretation has been possible, such a procedure was successfully applied.

In future works, two leads should be followed: the first one, dealing with industry stakes, would be to work on the full automation of this procedure; the second one, back to more fundamental research, would be to use the "early detection" method to provide more accurate depth values and bring even more confidence in the RGB-composite thermal D-scan.

\section{REFERENCES}

[1] Shepard S.M., Advances in pulsed thermography. Proc. of SPIE Thermosense XXIII, Vol. 4630, 511-515, 2001.

[2] Shepard S.M., Lhota J.R., Rubadeux B.A., Wang D. and Ahmed T., Reconstruction and enhancement of active thermographic image sequences. Optical Engineering, Vol. 42, No. 5, 1337-1342, 2003.

[3] Shepard S.M., Thermography in composites, Materials Evaluation, Vol. 65, No. 7, 690-696, 2007.

[4] Balageas D., Defense and illustration of time-resolved pulsed thermography for NDE. Quantitative InfraRed Thermography Journal, Vol. 9, No. 1, 3-32, 2012.

[5] Balageas D., Roche J.-M., Leroy F.-H., Les images de coefficients de la méthode TSR : un moyen simple et efficace de détecter et imager les défauts. Congrès de la Société Française de Thermique, Gérardmer, France, May 28-31, 2013.

[6] Roche J.-M., Leroy F.-H., Balageas D., Images of Thermographic Signal Reconstruction coefficients: a simple way for a rapid and efficient detection of discontinuities. Materials Evaluation, Vol. 72, No. 1, 73-82, 2014.

[7] Roche J.-M., Leroy F.-H., Balageas D., Information condensation in defect detection using TSR coefficients images. QIRT 2014, Bordeaux, July 7-11, 2014.

[8] Roche J.-M., Balageas D., Common tools for quantitative pulse and step-heating thermography - Part II: experimental validation. QIRT 2014, Bordeaux, July 7-11, 2014.

[9] Cielo P., Analysis of pulse thermal inspection. Proc. of the $14^{\text {th }}$ Symposium on NDE, S.W. Moore and G.A. Matzkanin ed., SRI/NTIAC, San Antonio, Texas, USA, 418-428, 1983.

[10] Vavilov V., Thermofit pro operation manual. Innovation, Ltd., 2000.

[11] Grinzato E., Temperature monitors works of art health as human beings. Proc. of the $16^{\text {th }}$ World Conference on Nondestructive Testing, Montreal, Canada, 2004.

[12] Dumoulin J., Ibos L., Marchetti M., Mazioud A., Detection on non-emergent defects in asphalt pavement samples by long pulse and phase infrared thermography. European Journal of Environmental and Civil Engineering, Vol. 15, 557-574, 2011.

[13] Vavilov V., Shirayev V.V., Thermal tomography. USSR Patent No. 1266308, 1984.

[14] Vavilov V., Thermal non destructive testing: short history and state-of-art. Proc. QIRT 1992 Conf., pp 179-194. Available at QIRT Open Archives: http://www.qirt.org/dynamique/index.php?idD=55 - Paper QIRT 1992-028.

[15] Swiderski W., The characterization of defects in multi-layered composite materials by thermal tomography methods. Acta Physica Polonica A(2009); 115(4): 800-8004.

Available at: $h$ ttp://yadda.icm.edu.pl/przyrbwn/element/bwmeta1.element.bwnjournal-article-appv115n410kz.

[16] Balageas D., Déom A., Bosher D., Contrôle non destructif des composites carbone-époxy par méthode photothermique impulsionnelle. Revue Générale de Thermique, No. 301, pp. 37-41, 1987.

[17] Balageas D.L., Déom A.A., Bosher D.M. Characterisation and non-destructive testing of carbon-epoxy composites by a pulsed photothermal method. Materials Evaluation, 45(4), 461-465, 1987.

[18] Bontaz J., Une méthode photothermique impulsionnelle appliquée au contrôle de matériaux composites (A pulse photothermal method applied to non-destructive testing of composite materials). Ph.D. thesis, University of Bordeaux, 1991.

[19] Bontaz J., Fort Ch., Horbette B., Identification de la profondeur et de la valeur de la résistance thermique de contact dans des matériaux stratifiés par la méthode photothermique impulsionnelle (identification of depth and contact thermal resistance in layered materials using the pulse photothermal method). Proc. Annual Conf. of the SFT (Soc. Franç. des Thermiciens), pp. 221-224, Nantes, May 17-19, 1990.

[20] Krapez J.-C., Balageas D., Early detection of thermal contrast in pulsed stimulated infrared thermography. Proc. QIRT 1994 Conf., pp 260-266. Available at QIRT Open Arch.: http://www.qirt.org/dynamique/index.php?idD=56 Paper QIRT 1994-039.

[21] Krapez J.-C., Balageas D, Déom A, Lepoutre F. Early detection by stimulated infrared thermography. Comparison with ultrasonics and holo/shearography. In: Maldague XPV, editor. Advances in signal processing for nondestructive evaluation of materials, NATO ASI series E. Vol. 262. Kluwer Acad. Publ; p. 303-321, 1994.

[22] Balageas D., In search of early time: an original approach in the thermographic identification of thermophysical properties and defects. Advances in Optical Technologies, article ID \#314906, 2013.

[23] Balageas D., Krapez J.-C., Cielo P., Pulsed photothermal modeling of layered material. Journal of Applied Physics, Vol. 59, No. 2, 348-357, 1986.

[24] Trousset E., Rannou J., Laruin F., Guillaumat L., Maire J.-F., Towards the assessment of low-velocity impact induced damage in laminate composite plates. Proc. of the $15^{\text {th }}$ European Conference on Composite Materials, Venice, Italy, 2012. 